\title{
DIFFERENCES AND SIMILARITIES BETWEEN STRUCTURAL PROPERTIES OF GAN GROWN BY DIFFERENT GROWTH METHODS
}

\author{
Z. Liliental-Weber, J. Jasinski and J. Washburn \\ Lawrence Berkeley National Laboratory 62/203, Berkeley, CA 94720
}

\begin{abstract}
In this paper defects formed in GaN grown by different methods are reviewed. The crystal growth direction and growth rate play important roles. For bulk crystals grown under high pressure the highest growth rates are for planes perpendicular to the c-axis. Only planar defects formed on c-planes are observed in these crystals. There are no threading dislocations or nanotubes in the c-direction. However, polarity of the growth direction plays a role in the surface roughness and the distribution of planar defects. For growth of homo-epitaxial and hetero-epitaxial layers the growth is forced to take place in the much slower c-direction. As a result defects related to the purity of constituents used for growth are formed such as nanotubes and pinholes. In addition threading dislocations and dislocations that accommodate lattice and thermal expansion mismatch are formed.
\end{abstract}

\section{Introduction}

In order to control defect formation, it is essential to understand the mechanisms of formation and the detailed structure of particular defects. In this paper characteristic defects in GaN grown heteroepitaxially on $\mathrm{Al}_{2} \mathrm{O}_{3}$ or $\mathrm{SiC}$ using different methods (MOCVD, HVPE and MBE) are compared to those found in bulk GaN crystals (grown from a dilute solution of atomic nitrogen in liquid gallium in the temperature range $1500 \mathrm{~K}-1800 \mathrm{~K}$ under high nitrogen pressure up to 20 kbars [1]). Defects formed in layers grown by MOCVD and HVPE on these bulk substrates will also be discussed.

\section{Defects in the MOCVD grown layers}

GaN layers were grown either on $\mathrm{SiC}$ or $\mathrm{Al}_{2} \mathrm{O}_{3}$ (sapphire) substrates using the MOCVD method. Usually a two step growth was applied with growth of a buffer layer (mostly AIN for growth on SiC and low-temperature $\mathrm{GaN}$ for growth on sapphire), followed by the main layer grown at higher temperature. The growth usually starts as islands which eventually coalesce to form a continuous layer [2]. Many dislocation half-loops are observed in the buffer layer and in the main layer in the area just above the buffer and the density of dislocations decreases when the layer is grown to greater thickness. The main defects observed in such layers are dislocations, nanotubes, pinholes and occasionally inversion domains (Fig. 1a-d). The majority of dislocations are edge type with Burgers vectors at $90^{\circ}$ to the c-axis.

It was observed that pinholes and nanotubes start from a V-defect (Fig. 1a-d) formed on (1011) planes with about $56^{\circ}$ between the $\mathrm{V}$ arms (see Fig. 1d), but only some of them develop into long empty tubes which either extend to the sample surface or terminate within the layer (Fig. 1a,b). Both types of defects appear to have the same origin, a nanotube may develop from what is originally a pinhole and extend for long distances along the c-axis. It is believed that these defects are impurity related [3].

\section{Defects in HVPE layers grown on sapphire}

Growth of GaN using HVPE is much faster than the growth using the MOCVD method, therefore, greater layer thickness can be grown in shorter time. By growing thicker layers more interaction between dislocations can take place which leads to a smaller density of dislocations near the top surface. Interaction between dislocations takes place at the greatest rate in the area close to the interface with the substrate where more dislocations with inclined line directions to the c-axis are present. As the layer becomes thicker there are fewer interactions between dislocations since they are further apart and decrease of dislocation density becomes more difficult. 

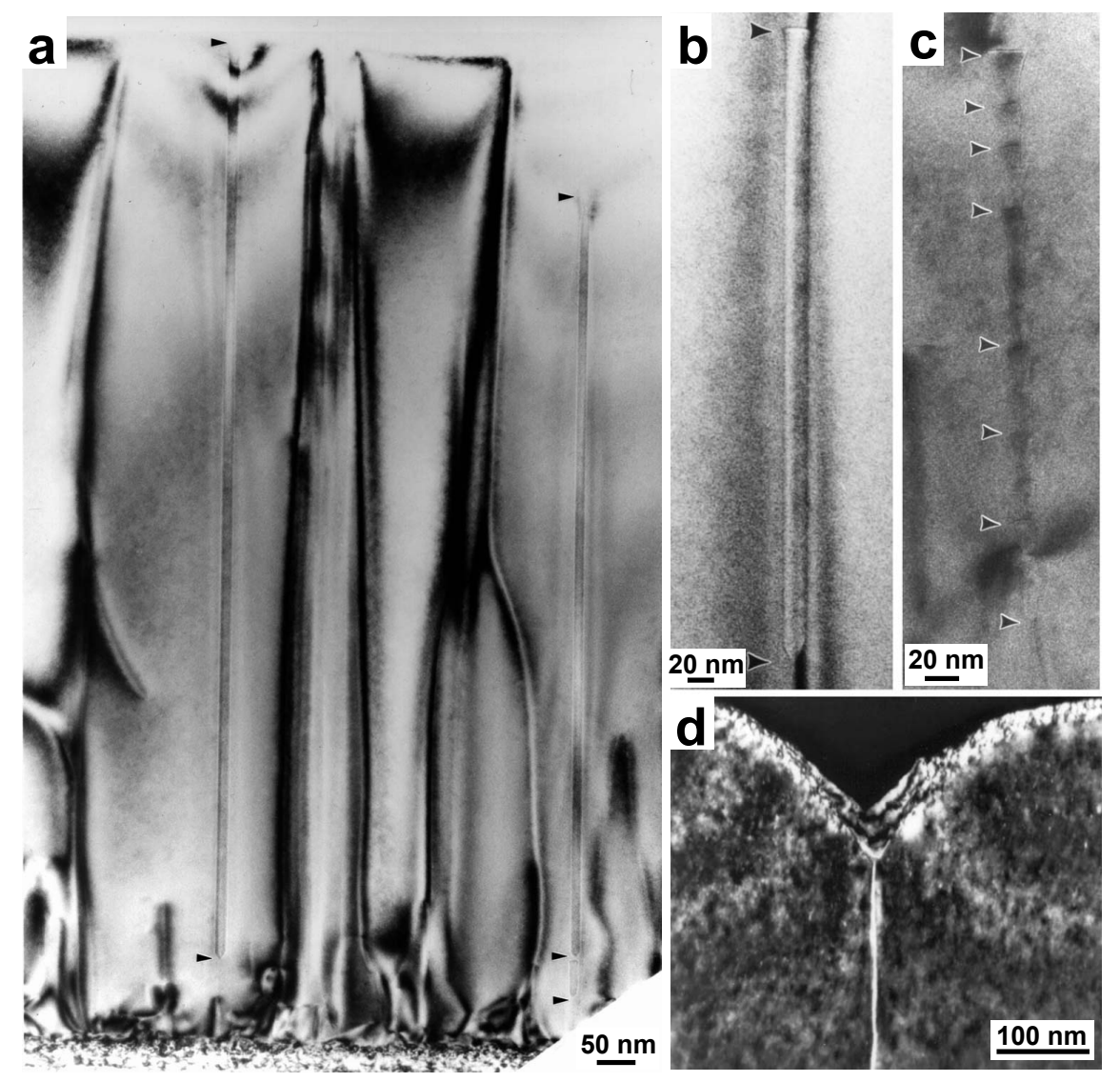

Fig. 1. (a) Defects (nanotubes, indicated by arrows, and dislocations) in a GaN layer grown on SiC by MOCVD with Ga polarity. Note that nanotube on the left hand-side propagates to the sample's surface and the one on the right hand-side is terminated inside the layer; (b) a nanotube along a screw dislocation terminated inside the GaN layer grown on $\mathrm{SiO}_{2}$. A nanotube starts from a $\mathrm{V}$-shape defect and then elongates in the c-direction with walls on (1100) planes; (c) pinholes with V-shape stacked on top of each other along a screw dislocation found in the MOCVD GaN layer grown on an $\mathrm{Al}_{2} \mathrm{O}_{3}$ substrate; (d) a pinhole attached to a screw dislocation formed at the surface of the same GaN layer.

Our studies showed that for layer thickness up to $300 \mu \mathrm{m}$ dislocation density has an inverse proportionality to film thickness [4]. A high number of pinholes (voids) seen in cross-sectioned samples as triangles aligned along screw dislocations, forming a bamboo-like structure, was found in the HVPE layers (Fig.. 2a). In plan-view configuration these voids had a hexagonal shape (Fig. 3a). Their formation can be related to the core structure of a screw dislocation. In order to obtain information about dislocation core structures, high-resolution focal series were used together with a numerical reconstruction procedure to reconstitute the electron wave at the specimen exit surface. For screw dislocations accompanied by voids in HVPE samples, such reconstructions were obtained for [0001] projection where atomic columns separated by $1.84 \AA$ can be clearly seen (Figs. 3 b-d). In the dislocation core, one atomic column at the expected tip of the V-type defect is very weak (Fig. 3c-area A) and another column in the dislocation core is very bright (Fig. 3c-area B). Our calculations show that weaker or stronger intensity of such columns can not be obtained simply by sample tilt, therefore such a difference in their intensity can be assigned only to the stoichiometry of the particular column. Weaker intensity at the tip of the Vdefect confirms our earlier suggestion that these defects are due to impurity accumulation on the low growth (1011) planes [3]. No indication of a continuous hollow core screw dislocation was obtained based on this study. 


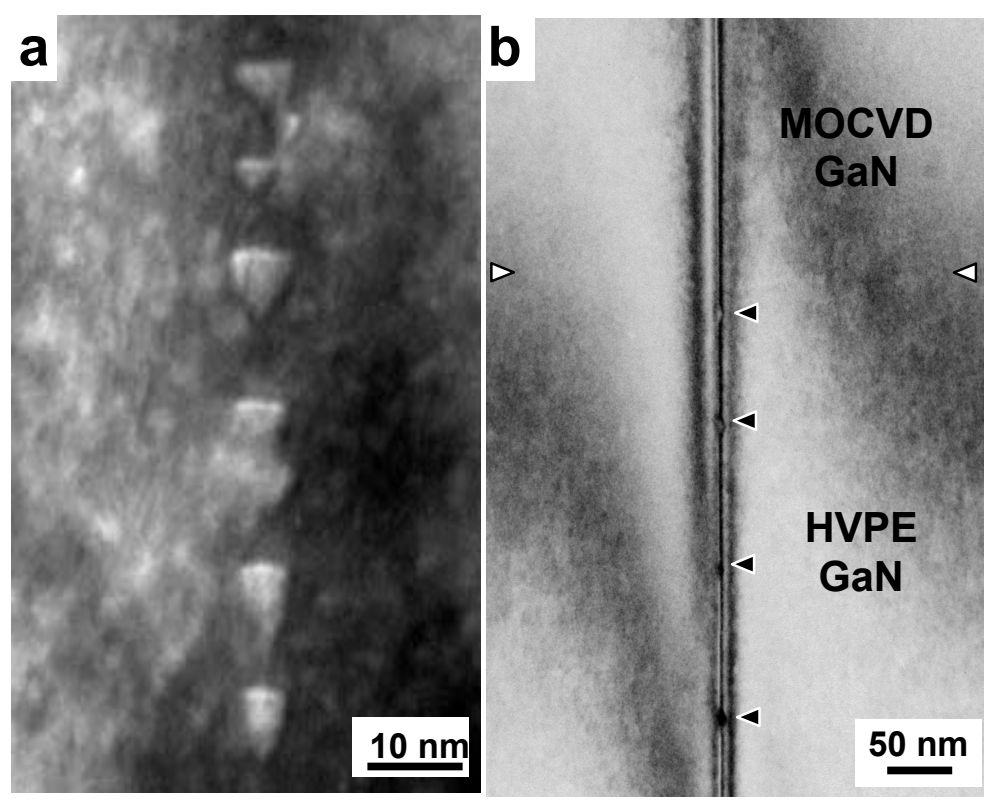

Fig. 2. (a) Pinholes along a screw dislocation in a HVPE GaN layer. Contrast on the dislocation disappears for this diffraction condition; (b) pinholes along a screw dislocation in the HVPE layer and lack of them in the layer grown on top of it by the MOCVD method. The image is taken with a diffraction condition for which the screw dislocation is in contrast.
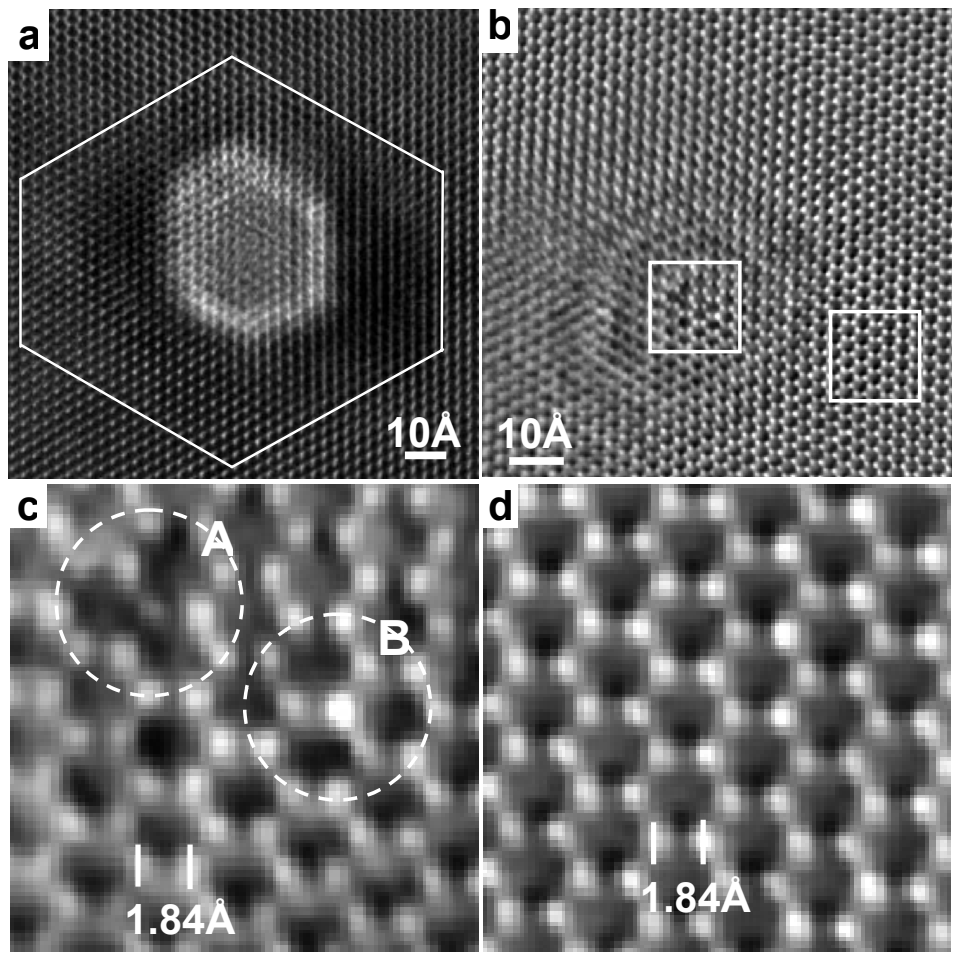

Fig 3. (a) Burgers circuits around a screw dislocation from the HVPE grown GaN layer; (b) phase of the electron wave reconstruction from the screw dislocation surrounded by a void; (c) larger magnification of defect area outlined by a square. A- reconstruction of the dislocation core structure around the tip of the void and B around the dislocation core; (d) reconstruction in the perfect material. Note that all atomic columns are preserved (as in the perfect material) suggesting that a hollow dislocation core is not formed. The intensity of atomic columns is weaker in the vicinity of the tip of the $\mathrm{V}$-shape void suggesting light elements present in the column. 


\section{Defects in MBE and MOCVD layers grown on top of HVPE layers}

Defects formed in Ga-rich MBE layers grown on top of HVPE layers showed that dislocations present in the HVPE layer propagate into the newly grown MBE layer. Voids were not formed along screw dislocations in the MBE layers grown with excess Ga despite the fact that Ga droplets were observed on the layer surface as well as some imbedded during growth. This suggests that these MBE layers had less impurity content compared to the HVPE growth. Similar observations were made for layers grown by MOCVD on the HVPE substrates. Dislocations from the underneath layer continue to propagate into the top layer. Despite the fact that pinholes were present along screw dislocations in the HVPE layer that they were not found along the dislocations in the MOCVD layer grown on top of it (Fig. 2b). Direct reconstruction of the phase and amplitude of the scattered electron wave from a focal series of high resolution images is in progress to determine the difference between core structure of screw dislocations in the HVPE layer and in the Ga-rich MBE layers.

\section{Bulk GaN crystals}

Bulk GaN is grown from a solution of atomic nitrogen in liquid gallium under high nitrogen pressure (up to $20 \mathrm{kbars}$ ) in the temperature range $1500-1800 \mathrm{~K}$ [1]. These crystals crystallized with a wurtzite structure in the form of platelets which have the shape of elongated hexagons [5]. The longest axis was usually along [1120]. In some cases the samples were less elongated and the dimensions along [1120] and [1100] were similar, but in all cases the smallest dimension was in the c-axis direction. The ratio of plate length to thickness along the $\mathrm{c}$ axis was typically as large as 100 . This shows that growth in the $\mathrm{c}$ axis direction is the most difficult. The largest platelets have a size close to $1 \mathrm{~cm}$. These crystals are polar and the growth polarity influenced the surface morphology. The surface of the plate with $\mathrm{N}$ polarity had a smooth surface while the opposite side was rough (Fig. 4).

The crystal near the side of the plate with the flat surface was structurally perfect, with no extended defects present. Many planar defects were observed close to the rough surface (Fig. 5a). These planar defects were stacking faults and dislocation loops decorated by Ga precipitates (Fig. 5b). These stacking faults probably nucleated during crystal growth by growth mistakes.

Growth of bulk GaN platelets takes place mainly at right angles to the c-axis, therefore planar defects formed on the c-planes are the most typical defects in this material. These defects do not propagate to epitaxial layers grown on top of them. Pinholes and nanotubes are not formed in this material since the fast growth along cplanes prevents their formation.

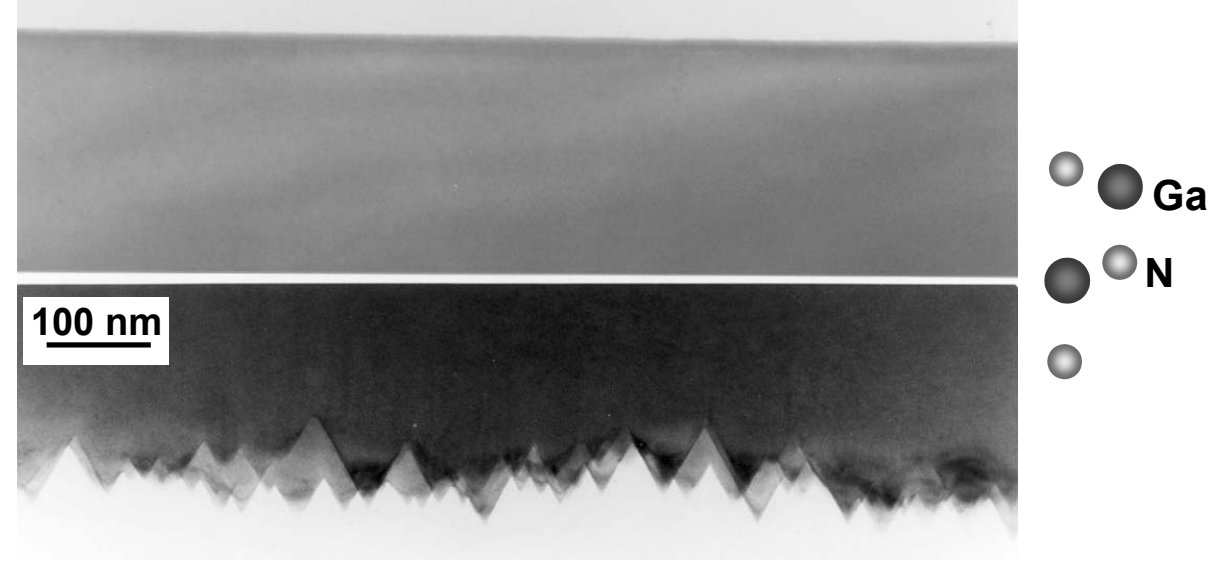

Fig. 4. A cross-section micrograph through a bulk GaN platelet showing the different surface roughness for opposite growth polarity (indicated on the right). High densities of planar defects (stacking faults and dislocation loops decorated by Ga precipitates) were found near the rough side of the platelet.

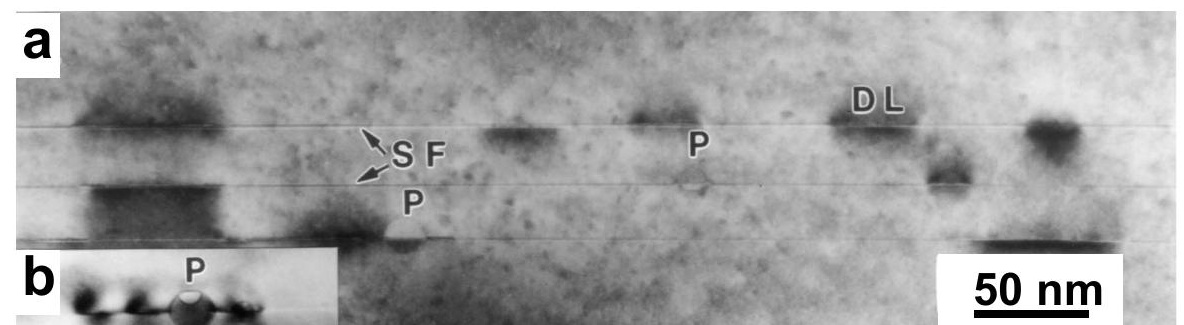


Fig. 5. (a) Stacking faults (SF) and dislocation loops (DL) decorated by Ga precipitates (P) were found on the rough side of the platelet; (b) dislocation loop observed edge-on with Ga precipitae $(\mathrm{P})$. Note a small void attached to the Ga precipitate at the upper side of the precipitate.

\section{Defects in MOCVD layers grown on bulk crystals}

TEM studies of the samples grown directly on bulk GaN platelets without a buffer layer showed that MOCVD epitaxial layers retain the polarity of the substrate [6]. However, growth on the two opposite c-surfaces (different polarity) is not equivalent from the point of view of defects in the homoepitaxial layers. In the epi-layer grown on the "smooth" non-polished surface (N-polarity) threading dislocations and inversion domains were found (Fig. 6a). These threading dislocations started either from dislocation loops formed at the interface or from some small "surface disturbances" from which several threading dislocations originated. Pinholes with walls on (1011) planes at the top of inversion domains were also observed. Since in this case growth is forced to be along the c-axis, which is much slower than growth in the perpendicular direction, impurities have enough time to accumulate on (1011) polar planes. It has been found that a N-polar surface decomposes faster and initiation of the growth has to be done at lower temperature than in the case of growth with Ga-polarity. This may explain why dislocation loops were formed at the interface which resulted in the formation of threading dislocations and inversion domains.

For the layer grown on the rough surface (Ga-polarity) no threading dislocations were observed. The interface between the substrate and the layer was not easily visible suggesting a very good continuation of growth in the layer [6]. However, a high density of pinholes $\left(\sim 10^{6}-10^{7} \mathrm{~cm}^{-2}\right)$ was observed.

\section{Defects in HVPE layers grown on bulk crystals}

First results for growth of epitaxial layers on bulk GaN using the HVPE technique have been obtained (Fig. 6b). Bulk GaN substrates were mechanically polished and etched by Reactive Ion Etching prior to the growth. For growth with both polarities abrupt interfaces were observed. No threading dislocations were present in these layers. The big difference was in the growth rate for the two polarities and in the surface smoothness. Growth with Ga polarity was three times faster than growth with $\mathrm{N}$ polarity, however, in the later case the surface was more smooth. Formation of some dislocation loops for growth with $\mathrm{N}$ polarity was observed, as was the case for growth using the MOCVD technique.

\section{Summary and Conclusions}

This review shows that the defects formed in GaN depend primarily on the crystallographic growth direction and on the growth polarity; this applies for all growth methods. For bulk crystals however, where growth proceeds primarily along [1120] and [1100] directions, planar defects on c-planes dominate. In bulk crystals caxis threading dislocation and nanotubes were not found, but surface morphology changed drastically for opposite growth polarities.

When homoepitaxial layers are grown by MOCVD or HVPE on these bulk substrates, the crystal is forced to grow in the c-axis direction which is several times slower. Depending on the growth polarity, growth of such a layer can be quite different. Impurities, substrate surface inhomogeneities or substrate decomposition can lead to the formation of threading dislocations, inversion domains, nanotubes and pinholes, similar to those observed in heteroepitaxial layers. Density of these defects in homoepitaxial layers can be negligible for well 
prepared substrate surfaces, but locally these defects may appear if any inhomogeneities (such as polishing damage-not shown in this paper) is present.
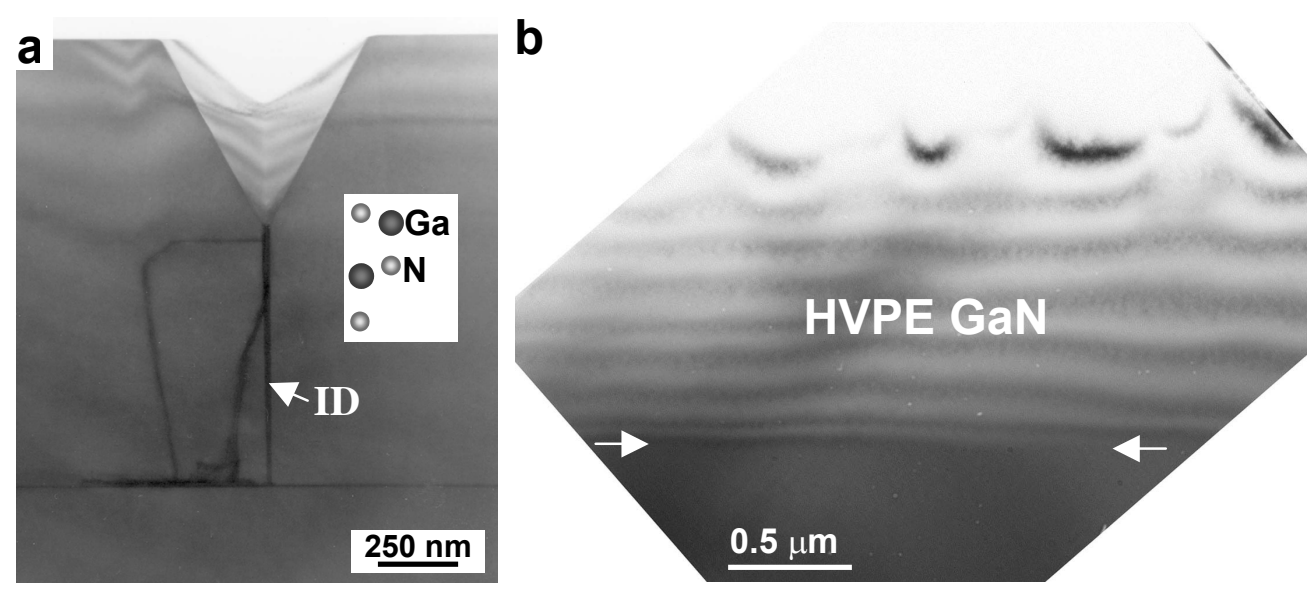

Fig. 6. (a) An inversion domain (ID) with a pinhole formed on top of it and a threading dislocation attracted to the pinhole formed in a MOCVD GaN layer grown with N-polarity on a bulk GaN substrate. All these defects started from the dislocation loop (darker contrast observed at the interface). A continuous dark line at the interface between the substrate and the epilayer indicates the presence of some types of inhomogeneity; (b) GaN layer grown also with N- polarity by HVPE on a bulk GaN substrate, first polished and etched by reactive ion etching.

In heteroepitaxial films, due to growth on substrates with different lattice parameter and different thermal expansion coefficient than the layer, a high density of misfit dislocations are formed at the interface, in addition to $t$ defects caused by impurities (nanotubes and pinholes) or surface damage. Since HVPE growth allows larger layer thickness, more interaction between threading dislocations can take place leading to substantial decrease of threading dislocation density at the top surface in thick layers. However, crystals grown by this method will never be dislocation free.

\section{Acknowledgment}

This work was supported by the U.S. Department of Energy under the contract No. DE-AC0376SF00098. The use of the facility at the National Center for Electron Microscopy at the LBNL is greatly appreciated. The authors want to thank Drs. S. Porowski, I. Grzegory, J. Baranowski, R. Dupuis, R. Molnar, H. Morkoc and S.S. Park for providing GaN samples.

\section{References:}

1. I. Grzegory, J. Jun, M. Bockowski, St. Krukowski, M. Wroblewski, B. Lucznik and S. Porowski, J. Phys. Chem. Solids 56, 639 (1995).

2. S. Ruvimov, Z. Liliental-Weber, H. Amano, and I. Akasaki, MRS Proc. vol.482, 387 (1998).

3. Z. Liliental-Weber, Y. Chen, S. Ruvimov, and J. Washburn, Phys. Rev. Lett. 79, 2835 (1997).

4. J. Jasinski and Z. Liliental-Weber, J. Electr. Mat. 31, 429 (2002).

5. Z. Liliental-Weber, C. Kisielowski, S. Ruvimov, Y. Chen, J. Washburn, I. Grzegory, M. Bockowski, J. Jun, and S. Porowski, J. Electr. Mat. vol. 25, 1545 (1996).

6. J.M. Baranowski, Z. Liliental-Weber, K. Korona, K. Pakula, R. Stepniewski, A. Wysmolek, I. Grzegory, G. Novak, S. Porowski, B. Monemar, and P. Bergman, Mater. Res. Soc. Symp. vol. 449, 393 (1997). 